\title{
Effect of erbium nanoparticles on structural and spectroscopic properties of bio- silica borotellurite glasses containing silver oxide
}

\begin{abstract}
$\mathrm{Er}^{3+} \mathrm{NPs}$ doped bio-silica borotellurite glasses containing silver oxide were prepared using melt quenching techniques. The silica used was extracted from rice husk by acid leaching process. Optical absorption and photoluminescence properties of the glass samples were studied. The samples were confirmed to be amorphous in nature from the XRD results. The FTIR results reveal the existing functional groups in the glass structure. XRF result shows that $98.6 \%$ silica was obtained from the rice husk. The optical absorption spectra revealed that fundamental absorption edge shifts to longer wavelength as the content of erbium NPs increases. The value of band gap had been calculated and shown to be increased with an increase content of erbium NPs. The Urbach energy was shown to be linearly decreased with an increase in content of erbium NPs oxides. The introduction of silver oxides modifies the optical properties of the glass. The emission cross-section $(\delta \mathrm{e})$ and gain bandwidth $(\mathrm{FWHM} \times \delta \mathrm{e})$ for ${ }^{4} I_{13 / 2} \rightarrow{ }^{4} I_{15 / 2}$ transition were calculated from the McCumber theory. The photoluminescence spectra revealed three emission bands corresponding to ${ }^{4} \mathrm{H}_{11 / 2}$ ${ }^{4} \mathrm{I}_{15 / 2}(470 \mathrm{~nm}),{ }^{4} \mathrm{~S}_{3 / 2}-{ }^{4} \mathrm{I}_{15 / 2}(561 \mathrm{~nm})$ and ${ }^{4} \mathrm{~F}_{9 / 2} \rightarrow{ }^{4} \mathrm{I}_{15 / 2}(758 \mathrm{~nm})$ transitions assigned to green and red emissions respectively. The results suggest that these glasses might be used as a promising candidate as well as gain media for solid state lasers .
\end{abstract}

Keyword: Erbium nanoparticles; Optical gain; McCumber theory; Near infrared spectrum 
